\title{
On the Choice of Parameter Values for Simulation Based Experiments on Mobile Ad Hoc Networks
}

\author{
Saher S. Manaseer \\ Department of Computer Science, The University of Jordan, Amman, Jordan \\ Email: saher@ju.edu.jo
}

Received 2 March 2016; accepted 23 April 2016; published 28 April 2016

Copyright @ 2016 by author and Scientific Research Publishing Inc.

This work is licensed under the Creative Commons Attribution International License (CC BY).

http://creativecommons.org/licenses/by/4.0/

(c) (i) Open Access

\begin{abstract}
Over the course of the last 10 years, research on Mobile Ad Hoc Networks has witnessed high levels of focus. Because of this field being a relatively new topic, most researchers have focused on using simulation as the first choice for performance measurement and evaluation. In order to contribute to the credibility and reliability of research results on Ad Hoc networks, this paper addresses the issue of choosing parameter values that are used as input for simulators used in such experiments by surveying a sample of recently published papers on Ad Hoc Networks. Results show that in the majority of revised papers, the simulation environment is not carefully planned as reflected by the diversity and quality of parameters used.
\end{abstract}

\section{Keywords}

\section{Simulation, Parameters, Ad Hoc Networks}

\section{Introduction}

Choosing the parameter values is one of the most essential steps of the experiment setup and design stage of any research [1]. The "correctness" of the values selected is a relatively broad term. For instance, a correct parameter could be defined as the more realistic one. On the other hand, a correct parameter set could be the group of parameters that accomplish the goal of simulation with the minimum cost [2].

In the field of MANETs, the sparse and none fully-explored space of parameter values can easily cause any researcher to incorrectly assign values to parameters used as input to the simulator. Moreover, the interrelations between different factors that affect the network performance are not fully understood. Moreover, the full list of factors is not fully discovered [3]. For example, when choosing the network size expressed in number of nodes, 
the researcher cannot certainly report that the selected number of nodes is suitable for the problem addressed. Moreover, the selected number of node is not definitely going to affect the results of simulation and, even if it does, the effect is neither predictable nor correctly related to other simulation parameter.

It is an extra step that needs to be taken by the researcher to justify the choices of parameters made. Manaseer [4] has shown some justification for the choice of simulation time and transmission range in relation to network area.

\section{Related Wok}

There have been many surveys in the last decade. Cavin et al. [2] have reported some issue related to the accuracy of simulations used in studying some flooding algorithm in MANETs. This paper has pointed out some issues related to the parameters. In 2005, Stuart Kurkowski et al. [1] performed a survey on a population of papers published between 2000 and 2005. Many pitfalls have been reported and they even are repeated in this work which is 10 years after the last survey was held. According to [1], 15\% had repeated work and only as little as $12 \%$ mention the simulator version used. The survey also has reported that only $7 \%$ of the surveyed papers have addressed some special issues such as initialization bias or random number generator issues.

The major observation at this point is that the recommendations and conclusions of survey papers, such as this one, are not being implemented when new research is conducted. Therefore, these surveys should continue.

\section{Simulation Related Issues}

\subsection{Realistic vs. Hypothetical}

Simulation as a tool has the advantage of testing a wide range of scenarios [5], including the ones that do not exist in real life. However, this ability is accompanied by major downsides. In many cases, researchers tend to choose the setup in a way that serves testing a new protocol without taking real life scenarios into account. It is relatively easy to build a none-realistic scenario that has no real-life counterpart represented by the chosen parameter set. Although this scenario can be used to test any protocol and help researchers to measure the performance of any new proposed solution, the main point of a new solution is to be used in real life applications in order for it to be of use.

\subsection{Relation between Parameters and Research Problem}

One of the most common mistakes in simulation based experiments is addressing irrelevant parameters [6]. Generating different scenarios via altering the values of irrelevant parameters, which do not affect the problem for which the simulation is designed, is a main source of redundancy in the total number of runs for this simulator. Therefore it is important to address only the parameters that are either directly or indirectly related to the research problem.

\subsection{Interrelation between Parameters}

Computer networks have a large number of factors that might affect the overall performance levels. It is necessary to understand the interaction between these factors and to take this into account when designing the simulation scenarios. Moreover, special care should be taken when varying more than one parameter to ensure that the values chosen do not contradict with the relation that binds the two factors together if any [4].

\subsection{Performance Measurement}

In most of the available simulators, results are recorded and trace files [7]. Most of the trace files provide adequate level of details. However, it is essential to ensure that the details provided in the trace files enable the extraction of the intended performance measurements criteria that, in turn, have to be pre decided before conducting simulation.

\subsection{Justification of Choices Made}

Finally, to gain a higher level of credibility for any conducted simulation based research, it is necessary to provide an adequate level of justification for the values chosen into different scenarios used with the simulation. It 
is also a common mistake to follow a trial and error method to decide the best scenarios that have the highest level of performance. Using this approach can easily lead to presenting results for scenarios that do not actually reflect any realistic Applications.

\section{Survey}

In this section, a collection of published research articles are surveyed to investigate the points mentioned above in order to provide future research with a preliminary guide to produce more reliable research findings.

\subsection{Description}

In order to perform this survey, more than 190 research articles have been reviewed and summarized. The articles have been published over the last five years and they are published in peer reviewed journals. The full list of papers is available upon request. Review process focused on the simulation parameters used and the method of presentation the researchers used to show them. These papers have been classified into two groups; the first group contains the papers with no parameters provided. For the second group, the parameters having summarized and filtered to choose the set of most common parameters addressed.

\subsection{Average Number of Parameters Used}

The scenarios described in the papers of the study population have used widely variable topologies in which the number of parameters ranges between a few of parameters to a long redundant list. Figure 1 shows the average number of scenario parameters reported by the papers against the number of the occurrences.

As seen in the figure, the majority of papers have reported between 6 - 10 parameters when describing the scenarios. Figure 1 shows the number of parameters only and not the number of values per parameter. The greater numbers of parameters indicate that the paper is addressing very specific case to implement any proposed work. However, it is worth mentioning that listing small number of parameters leads to weak future comparisons with the proposed work while, on the other hand, an extremely large list of parameters leads to confusion when the audience try to understand the exact focus and scope of the proposed work. Using small number of parameters indicates that the authors of the paper focus on the proposed technique and its representation more than the implementation. The unsuitable number of parameters indicated lack of understanding of the simulation environment and its requirements.

\subsection{Most Used Parameters (Top 10)}

Literature does not provide a manual that instructs the use of a predefined list of parameters in simulations. According to the sample used in this survey, researchers focus on using some parameter more frequently than other parameters. Figure 2 displays the top 10 parameters used along with their usage percentages.

It is worth mentioning that the focus on a certain parameter does not necessarily mean that this parameter is suitable for use with most scenarios. It might simple mean that researchers copy the values used in literature.

\subsection{Number of Values Per Parameter}

Due to the sparse nature of MANETs, researchers tend to test using wide range of values per parameter. Table 1 shows that the distinct values of parameters are limited only for the parameters that have limited space of

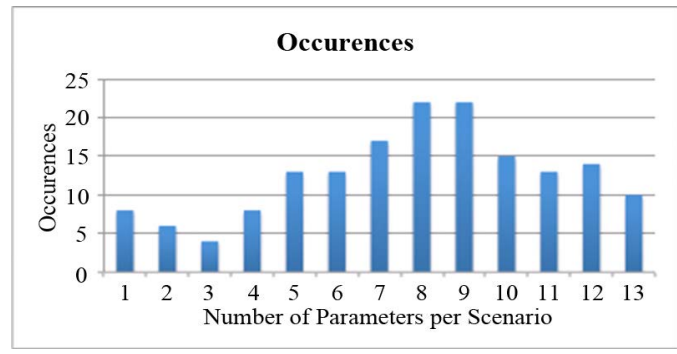

Figure 1. Occurrences of the parameter list size. 


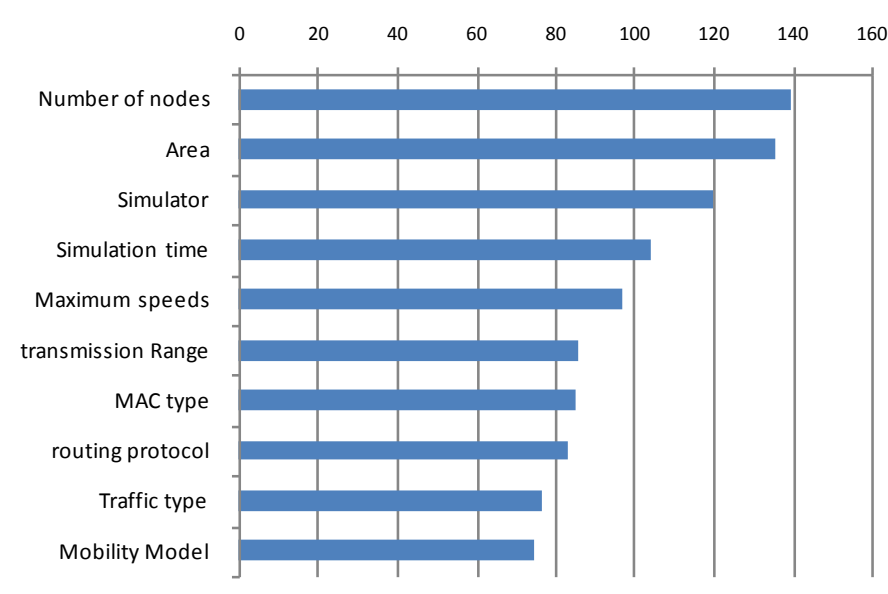

Figure 2. Top 10 used parameters.

Table 1. Different values per parameter.

\begin{tabular}{cccc}
\hline Paper number & Distinct values & Paper number & Distinct values \\
\hline Number of nodes & 80 & Packet Size & 22 \\
Maximum speeds & 72 & Mobility Model & 19 \\
Area & 64 & MAC type & 17 \\
Simulation time & 48 & Bandwidth & 15 \\
Routing protocol & 47 & Transmission interval & 14 \\
Simulator & 35 & Number of senders & 11 \\
Transmission Range & 34 & Radio-propagation & 11 \\
Pause time & 30 & Interface queue type & 8 \\
Packet rate & 23 & Numbers of Iterations & 8 \\
Data Rate & 22 & Antenna type & 6 \\
\hline
\end{tabular}

possible values. An example of such parameter is the antenna type for which the range of values is limited by the known types of antennas in real life.

The main effect of using a wide range of values per parameter is the loss of research focus. An extremely wide range of scenarios leads to difficulty in analyzing the results of the simulator. The large range of parameters values indicates the unawareness of the authors of the scenarios for which the proposed technique was developed.

\subsection{The Suitability of Values Chosen}

Among the choices made, there are two types of constraints that should be applied in any simulated research; the correlations between the parameters and the suitability of these parameters for use in real life scenarios. For example, taking two of the top three most common parameters used, Number of nodes and Network Area, the relation is never clearly stated as a justification. As seen in Figure 3, more than $50 \%$ of the surveyed papers use a ratio of $10 \%$ or less. This means that the density of the network has to be justified by the transmission range for example. Moreover, the figure also shows some non-realistic values where some scenarios have a ratio of $250 \%$. It can also be seen that approximately $25 \%$ of the papers studied have not considered the relation at the first place. This unplanned choice of values chosen shows the weakness of the performance prediction abilities about the functionality of the proposed mechanisms.

\subsection{The Choice of Simulator}

Figure 4 presents the percentages of usage for the major simulators. The most common Simulator is NS2. It is 


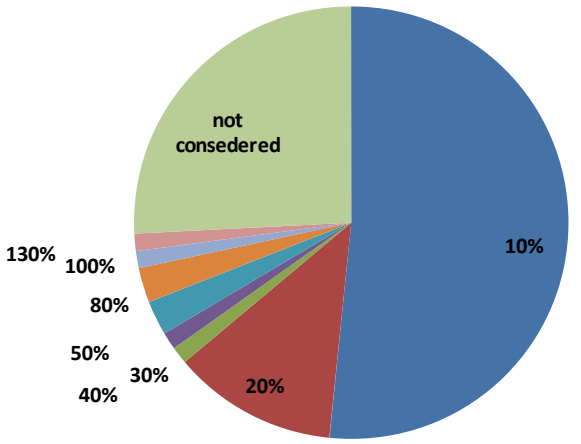

Figure 3. Node number to area ratio.

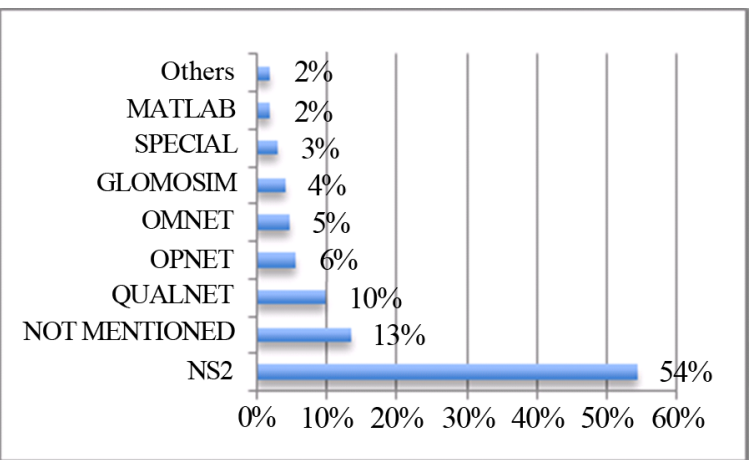

Figure 4. Simulator usage.

worth mentioning that the second place is filled with research papers that never mentioned the simulator choice at all. Choosing the simulation tool is a step that is affected by many reasons [5]. For instance, the cost of any simulator can remove it from the list. Moreover, some researchers tend to use some none common simulators or even self-developed simulators. Although this can be caused by lack of understanding of the common simulators, sometimes it might be triggered by the fact that the researcher needs to simulate specific small-scale improvements. Moreover, NS has a wide spread due to the facts that it is an open source and free simulator. Many researches are held in institutes where financial support is a major issue.

\section{Conclusions}

In this paper, we have surveyed a number of research papers, the results show that although many of the pitfalls reported here are reported in earlier surveys, they still exist. Moreover, it is clear that the experiment setup is still not at an optimal level. The reasons of such lack of optimality need further study. However, the lack of knowledge about the exact set of parameters and their values is clear in the findings of this survey.

This work shows that the nature of MANETs is still not fully explored. The exact list of effective parameters is not fully explored leading to tendency to simulate large number of scenarios to compensate the quality with quantity. In addition, the relations between the simulation environment parameters are not clear. This leads to generating multiple scenarios to assure the coverage of these ambiguous relations.

\section{References}

[1] Kurkowski, S., Camp, T. and Colagrosso, M. (2005) MANET Simulation Studies: The Incredibles. ACM SIGMOBILE Mobile Computing and Communications Review, 9, 50-61. http://dx.doi.org/10.1145/1096166.1096174

[2] Cavin, D., Sasson, Y. and Schiper, A. (2002) On the Accuracy of MANET Simulators. In Proceedings of the 2nd ACM International Workshop on Principles of Mobile Computing, Toulouse, 30-31 October 2002, 38-43. http://dx.doi.org/10.1145/584490.584499

[3] Andel, T.R. and Yasinsac, A. (2006) On the Credibility of Manet Simulations. Computer, 39, 48-54. http://dx.doi.org/10.1109/MC.2006.242 
[4] Manaseer, S. (2010) On Backoff Mechanisms for Wireless Mobile Ad Hoc Networks. Doctoral Dissertation, University of Glasgow, Glasgow.

[5] Weingärtner, E., Vom Lehn, H. and Wehrle, K. (2009) A Performance Comparison of Recent Network Simulators. 2009 IEEE International Conference on Communications, ICC’09, Dresden, 14-18 June 2009, 1-5.

[6] Engelbrecht, A.P., Cloete, I. and Zurada, J.M. (1995) Determining the Significance of Input Parameters Using Sensitivity Analysis. In: Mira, J. and Sandoval, F., Eds., From Natural to Artificial Neural Computation, Springer, Berlin Heidelberg, 382-388. http://dx.doi.org/10.1007/3-540-59497-3_199

[7] Issariyakul, T. and Hossain, E. (2011) Introduction to Network Simulator NS2. Springer Science \& Business Media. 


\section{Appendix A}

\section{Sample of Surveyed Papers}

- Ahmed, Firoz, and Hoon Oh. “An Encryption Based Black Hole Detection Mechanism in mobile Ad Hoc Networks.” International Journal of Security and its Applications 7.6 (2013): 1-10.

- Singh, JyotiPrakash, ParamarthaDutta, and AmlanChakrabarti. "Weighted Delay Prediction in Mobile ad Hoc Network Using Fuzzy Time Series.” Egyptian Informatics Journal 15.2 (2014): 105-114.

- Senthilkumaran, T., and V. Sankaranarayanan. "Dynamic Congestion Detection and Control Routing in Ad Hoc Networks.” Journal of King Saud University-Computer and Information Sciences 25.1 (2013): 25-34.

- Park, Seungjin, and Seong-Moo Yoo. “An Efficient Reliable One-Hop Broadcast in Mobile Ad Hoc Networks.” Ad Hoc Networks 11.1 (2013): 19-28.

- Oliveira, Rodolfo, et al. “Improving Path Duration in High Mobility Vehicular Ad Hoc Networks.” Ad Hoc Networks 11.1 (2013): 89-103.

- Carli, Marco, Stefano Panzieri, and Federica Pascucci. “A Joint Routing and Localization Algorithm for Emergency Scenario.” Ad Hoc Networks 13 (2014): 19-33.

- Zhao, Shushan, Robert Kent, and Akshai Aggarwal. “A Key Management and Secure Routing Integrated Framework for Mobile Ad-Hoc Networks.” Ad Hoc Networks 11.3 (2013): 1046-1061.

- Imran, Muhammad, et al. "Localized Algorithm for Segregation of Critical/non-Critical Nodes in Mobile ad Hoc and Sensor Networks.” Procedia Computer Science 19 (2013): 1167-1172.

- Budyal, V., and S. S. Manvi. “Intelligent Agent Based Delay Aware QoS Unicast Routing in Mobile Ad Hoc Networks.” International Journal of Multimedia and Ubiquitous Engineering 8.1 (2013): 11-28.

- Duran-Limon, H. A., et al. "A Network QoS Framework for Real-Time Event Systems in highly Mobile Ad-Hoc Environments.” Journal of Applied Research and Technology 12.3 (2014): 343-358.

- Gupta, Neha, and Manish Shrivastava. "Securing Routing Protocol by Distributed Key Management and Threshold Cryptography in Mobile Ad Hoc Network.” Int. J. Adv. Comput. Res 3.9 (2013): 13-18.

- Kishore, Nand, Sukhvir Singh, and RenuDhir. "Energy Based Evaluation of Routing Protocol for MANETs.” International Journal of Computer Science and Engineering (IJCSE) 2.3 (2014): 14-17.

- Gupta, Shailender, et al. “Towards Realistic Performance Evaluation of Delay Tolerant Network.” International Journal of Advanced Science and Technology 54.5 (2013): 39-48.

- Hussain, Syed A., and A. Saeed. “An Analysis of Simulators for Vehicular Ad Hoc Networks.” World Applied Sciences Journal 23.8 (2013): 1044-1048.

- Jaiswal, Siddhant, and D. S. Adane. "Hybrid Approach for Routing in Vehicular Ad-Hoc Network (VANET) Using Clustering Approach.” International Journal of Innovative Research in Computer and Communication Engineering, ISSN (2013): 2320-9798.

- Moravejosharieh, Amirhossein, et al. "Performance Analysis of AODV, AOMDV, DSR, DSDV Routing Protocols in Vehicular Ad Hoc Network.”Research Journal of Recent Sciences 2.7 (2013): 66-73.

- Wang, Dongbin, et al. “A Truthful and Low-Overhead Routing Protocol for Ad Hoc Networks.” International Journal of Future Generation Communication and Networking 6.2 (2013): 127-138.

- Chuang, Po-Jen, and Ting-Yi Chu. "MRBL: An Efficient Multicast Routing Protocol with Backup Labeling in MANETs." International Journal of Future Generation Communication and Networking 7.1 (2014): 125-136.

- Fernandez, E. M. G., et al. "Effect of Location Accuracy and Shadowing on the Probability of Non-Interfering Concurrent Transmissions in Cognitive Ad Hoc Networks.” Radioengineering (2013).

- Feng, Zuhong, Long Wang, and Xiujuan Gao. “An Improved Routing Protocol Ad-AODV Based on AODV.” International Conference on Information Science and Computer Applications (ISCA 2013) Published by Atlantis Press. 2013.

- Chanei, Waris, and SakunaCharoenpanyasak. "Enhanced Mobile Ad hoc Routing Protocol Using Cross Layer Design in Wireless Sensor Networks.”New Paradigms in Internet Computing. Springer Berlin Heidelberg, 2013. 1-11.

- Kim, Ki-Il. “A Simulation Study for Typical Routing Protocols in Aircraft Ad Hoc Networks.” International Journal of Software Engineering and Its Applications 7.2 (2013): 227-234.

- Kinalis, Athanasios, et al. "Biased Sink Mobility with Adaptive Stop Times for Low Latency Data Collection in Sensor Networks." Information fusion 15 (2014): 56-63.

- Banga, Sourabh, and Preeti Gera. "Simulation Study of Congestion Control on Ad-Hoc Wireless Sensor Network.” Journal of Applied Engineering (JOAE) 2.7 (2015).

- Para, Jyoti, and Laxmi Shrivastava. "Design and Implementation of Bellman, RIP and ZRP Routing Protocols of Mobile Ad-hoc Network (MANET).”IJCCER 2.3 (2014): 136-139.

- Bhatia, Tarunpreet, and A. K. Verma. "Simulation and Comparative Analysis of Single Path and Multipath Routing Protocol for MANET.” Anveshanam-The Journal of Computer Science \& Applications, vol. II 1 (2013). 


\section{Continued}

- Dhawan, Swati, and Vinod Saroha. “Optimize the Routing Protocol (GRP, OLSR, DSR) Using OPNET \& Its Performance Evaluation.” International Journal of Advances in Engineering \& Technology 6.3 (2013): 1399.

- Sethi, Srinivas, Sangita Pal, and Ashima Rout. "Effective Performance Evaluation of On-Demand Routing Protocol for Cognitive Radio Ad Hoc Network.” International Journal of Advanced Computer Research (IJACR) 3 (2013).

- Singh, Tejpreet, Balpreet Kaur, and Sandeep Kaur Dhanda. "Performance Evaluation of Routing Protocols in VANETs.” International Journal of Advanced Research in Computer and Communication Engineering 2.9 (2013).

- Haq, Furqan, and Thomas Kunz. “Simulation vs. Emulation: Evaluating Mobile Ad Hoc Network Routing Protocols.” Proceedings of the International Workshop on Wireless Ad-hoc Networks (IWWAN 2005). 2005.

- Sun, Haifeng. “Greedy Zone Epidemic Routing in Urban Vehicular Ad Hoc Networks.” (2013).

- Meghanathan, Natarajan, and Joseph Thompson. “On the Different Forms of Spanning Tree-Based Broadcast Topologies for Mobile Ad Hoc Networks.”International Journal of Combinatorial Optimization Problems and Informatics 4.1 (2013): 3.

- Qureshi, Kashif Naseer, Abdul Hanan Abdullah, and Rohana Yusof. "Position-based Routing Protocols of Vehicular Ad Hoc Networks \& Applicability in Typical Road Situation.” Life Science Journal 10.4 (2013).

- Islam, Md Manowarul, MdAbdur Razzaque, and Md Ashrafuddin. "Stable Route Development and Repairing Mechanisms for Mobile Ad Hoc Networks.” International Journal of Computing Communication and Networking Research 2.1 (2013).

- Macker, Joseph. "Mobile Ad Hoc Networking (MANET): Routing Protocol Performance Issues and Evaluation Considerations.” (1999).

- Sharma, Meenakshi, and Davinderjeet Singh. "Implementation of a Novel Technique for a Secure Route by Detection of Multiple Blackhole Nodes in Manet.” International Journal of Current Engineering and Technology E-ISSN (2014): 2277-4106.

- AL-Hilfi, Haider Mohammed Turki, et al. "Performance Comparison of a Reactive and Hierarchical Routing Protocol in 802.16 e Using Qualnet 6.1.”Terrain 1500 (2014): x1500.

- Hundal, Gagandeep Singh, Sunil Kumar Gupta, and Rajeev Bedi. “Adaptive Approach to find a Stable Path between Nodes in MANET.” Int J CurrEngTechnol 4.4 (2014): 2898-2901.

- Imaizumi, Naoya, et al. "Effective Flooding over Disaster Tolerant Ad Hoc Network based on Exchange of Neighbor Information." Proceedings of the International Conference on Parallel and Distributed Processing Techniques and Applications (PDPTA). The Steering Committee of the World Congress in Computer Science, Computer Engineering and Applied Computing (WorldComp), 2013.

- Brienza, Simone, et al. “A Learning-Based Algorithm for Optimal Mac Parameters Setting in IEEE 802.15. 4 Wireless Sensor Networks."Proceedings of the 10th ACM symposium on Performance Evaluation of Wireless Ad Hoc, Sensor, \& Ubiquitous Networks. ACM, 2013.

- Alwidian, Sanaa A., Ismail M. Ababneh, and Muneer O. Bani Yassein. "Neighborhood-based Route Discovery Protocols for Mobile Ad hoc Networks.” International Journal of Mobile Computing and Multimedia Communications (IJMCMC) 5.3 (2013): 68-87.

- Khiavi, Mina Vajed, Shahram Jamali, and Sajjad Jahanbakhsh Gudakahriz. "Performance Comparison of AODV and AOMDV Routing Protocols in Mobile Ad Hoc Networks.” International Research Journal of Applied and Basic Sciences (IRJABS) 4.11 (2013): 3277-3285. 